\title{
Rechtsverbindlichkeiten im OP-Bereich zu Beginn des neuen Millenniums
}

\author{
H. Hölzer, H. Striegel, C. Winter
}

\section{Zusammenfassung}

Bedingt durch die zunehmende Spezialisierung und Differenzierung der verschiedenen medizinischen Fachrichtungen entstehen unter rechtlicher Betrachtung erhöhte Anforderungen an Organisation, Koordination und Kommunikation der einzelnen Teilbereiche einer Klinik. Vor allem im Bereich der operativen Medizin zeigt sich, wie wichtig eine funktionsfähige Einheit aus Operationsteam, Anästhesie und weiteren beteiligten Fachabteilungen ist. Beim Einsatz neuer Technologien, insbesondere computergestützter Operationssysteme bestehen aufgrund des Studiencharakters der Versorgung erhöhte rechtliche Anforderungen in Bezug auf Aufklärung, Indikation, Durchführung und Koordination der Behandlung sowie hinsichtlich einer umfassenden Dokumentation. Daneben ergibt sich aus einer erhöhten Zahl nosokomialer Infektionen in operativen gegenüber nicht operativen Fachgebieten, dass besonders bei Operationen auf hygienische Maßnahmen und eine ausreichende Desinfektion geachtet werden muß. Die sich hieraus ergebenden Verantwortlichkeiten der ärztlichen und nichtärztlichen Mitarbeiter sowie des Klinikträgers in Bezug auf die Behandlung ihrer Patienten sollen im Folgenden dargestellt werden.

\section{Einleitung}

Arbeitsteilung ist aus der heutigen Medizin nicht mehr wegzudenken. Die Fortschritte der modernen Medizin beruhen ganz wesentlich auf einer ständig wachsenden Spezialisierung und Subspezialisierung der verschiedenen Fachrichtungen innerhalb der Medizin, die häufig eng zusammenarbeiten und sich gegenseitig ergänzen. Eine ähnliche Entwicklung zeigt sich im Bereich der nicht ärztlichen Heilberufe. Auch hier haben sich einzelne Fachrichtungen mit unterschiedlichster Spezialisierung herausgebildet, die in der modernen Medizin eine umfassende Patientenversorgung garantieren. Den großen Vorteilen, die eine arbeitsteilige Aufgabenwahrnehmung sowohl in horizontaler als auch in vertikaler Hinsicht mit sich bringt, stehen spezifische Gefahren im Hinblick auf Kommunikation, Kooperation und Koor-

OP-JOURNAL 2000; 16: 210-215

(c) Georg Thieme Verlag Stuttgart · New York dination der einzelnen Arbeitsabläufe gegenüber. Gerade an den Schnittstellen der geteilten Aufgabenerfüllung ergeben sich Fehlerquellen, die durch strukturelle Maßnahmen entschärft werden müssen. Aus juristischer Sicht ergibt sich bei geteilter Aufgabenerfüllung die Frage der Verantwortlichkeit im Falle eines Behandlungsfehlers. Welche diagnostischen, organisatorischen und therapeutischen Maßnahmen der Standard im Einzelfall fordert, kann u.a. anhand der - auch im Internet verbreiteten - Leitlinien der jeweiligen Fachgesellschaften festgestellt werden.

Der Einsatz von computerassistierten Systemen mit dem Ziel, Operationen präziser durchführen zu können, wird mittlerweile seit mehr als einem Jahrzehnt zunehmend intensiver betrieben, und inzwischen kann auch auf diesem Gebiet auf Erfolg versprechende Entwicklungen verwiesen werden. Der Einsatz neuer Technologien in der Medizin ist jedoch mit einer nicht auszuschließenden Gefahrerhöhung für den Patienten verbunden, da ex ante nicht alle Komplikations- möglichkeiten, die sich z.T. gerade aus dem Einsatz der neuen Technologien ergeben, vorhergesehen werden können. Aus dem z.T. noch experimentellen Charakter derartiger Verfahren ergeben sich gerade unter forensischen Gesichtspunkten besondere Anforderungen an Dokumentation, Aufklärung und organisatorische sowie technische Durchführung der Eingriffe, die tunlichst beachtet werden sollten, um die Haftungsrisiken zu minimieren.

Bei der Behandlung nosokomialer Infektionen ergeben sich durch die zunehmende Selektion resistenter Keime erhebliche Probleme, mit deren weiterer Zunahme in Zukunft gerechnet werden muss. In operativen Fachgebieten ist bei allen Eingriffsarten die überwiegende Zahl postoperativer Wundinfektionen auf eine endogene oder exogene Keimverschleppung während der Operation selbst zurückzuführen. Insoweit sind Maßnahmen der OP-Hygiene, Desinfektion und das Verhalten des OP-Personals selbst die entscheidenden prophylaktischen Schritte. Ganz abgesehen von dem aus postoperativen Wundinfektionen entstehenden volkswirtschaftlichen Schaden müssen auch rechtliche Konsequenzen bei Nichteinhaltung des medizinischen Standards in Bezug auf Hygiene und Desinfektion beachtet werden, wobei der forensische Schwerpunkt bei Infektionen in der Regel nicht auf dem Eingriff selbst, sondern der postoperativen Überwachung (zu spät erkannte Infektionen) liegt.

Problembereiche:

Arbeitsteilung - Einsatz neuer Technologien - Infektionen.

\section{Arbeitsteilung und Spezialisierung}

Im Fall der arbeitsteiligen Aufgabenwahrnehmung können sich für den Patienten insbesondere aus Irrtümern oder Missverständnissen zwischen den an der Behandlung beteiligten Personen, aus Koor- 
dinations- und Kommunikationsmängeln, aber auch aus einer unzureichenden Qualifikation oder Überwachung der zur Assistenz eingesetzten Mitarbeiter erhebliche Gefahren ergeben. Für die Frage der Verantwortlichkeit gegenüber dem Patienten ist zunächst zwischen horizontaler und vertikaler Arbeitsteilung zu unterscheiden.

\section{Horizontale Arbeitsteilung}

Horizontale Arbeitsteilung ist durch partnerschaftliche Gleichstellung der Beteiligten gekennzeichnet. Charakteristisch ist insbesondere die Weisungsfreiheit sowie ein sich entsprechender Ausbildungsstand der Beteiligten. Hierzu zählen Zusammenarbeit mehrerer Fachärzte gleicher (z.B. Chirurg - Chirurg) oder verschiedener Fachrichtungen eines Krankenhauses (z.B. Chirurg - Anästhesist), aber auch die Kooperation zwischen niedergelassenem Arzt und Klinikarzt. Speziell für das Verhältnis zwischen Chirurgen und Anästhesisten wurde durch den Berufsverband Deutscher Anästhesisten und dem Berufsverband der Deutschen Chirurgen eine Vereinbarung über die Zusammenarbeit bei der operativen Patientenversorgung abgeschlossen (vgl. MedR 1983, 21). Eine ähnliche Vereinbarung existiert auch zwischen Gynäkologen und Anästhesisten (vgl. Der Frauenarzt 1996, 1172 ff). Grundsätzlich hat jeder Arzt denjenigen Gefahren zu begegnen, die seinem Aufgabenbereich entstammen, d.h. der Operateur operationsspezifischen und der Anästhesist narkosespezifischen Gefahren. Er darf sich auch darauf verlassen, dass ein Kollege eines anderen Fachgebiets seine Aufgaben mit der gebotenen Sorgfalt erfüllt. Jedoch hat er dessen Angaben auf deren Plausibilität hin zu überprüfen. Treten offensichtliche Fehlleistungen oder Qualitätsmängel auf, so muss - unabhängig davon, in wessen Fachgebiet sie entstanden sind - der Arzt bzw. das nicht ärztliche Personal, das den Mangel erkennt, für die Mängelbeseitigung sorgen.

Horizontale Arbeitsteilung: Beispiele: Chirurg - Chirurg, Chirurg - Anästhesist. Haftung: jeder für seinen Bereich, außer bei offensichtlichen Fehlern.

Der dargestellte Vertrauensgrundsatz gilt jedoch nur dann, wenn es um Risiken geht, die ausschließlich dem Aufgabenbereich eines der beteiligten Mediziner zugeordnet werden können. Ergeben sich die Risiken für den Patienten aber gerade aus dem Zusammenwirken der an der Heilmaßnahme beteiligten Ärzte, so haben beide die Risiken auszuschließen, die sich aus der Unverträglichkeit der von den Beteiligten vorgesehenen Methoden ergeben könnten. Dies gilt insbesondere dann, wenn sich Gefahren verwirklichen, die dem von den Ärzten voll beherrschbaren Gefahrenbereich entstammen. Der Bundesgerichtshof entschied hierzu, dass für Verbrennungen im Gesicht der Patientin, die durch eine Explosion bei Zufuhr von reinem Sauerstoff im Rahmen der Narkose und gleichzeitigem Thermokautern bei einer Augenoperation entstanden sind, sowohl Operateur als auch Anästhesist haften.

\section{Vertikale Arbeitsteilung}

Vertikale Arbeitsteilung ist durch ein mit Weisungsrechten verbundenes Subordinationsverhältnis charakterisiert, das üblicherweise im Verhältnis von Chefarzt zu Oberarzt zu Assistenzarzt anzunehmen ist. Auch im Verhältnis von ärztlichem zu nicht ärztlichem Personal kann ein Subordinationsverhältnis vorliegen, wobei allerdings beachtet werden muss, dass Ärzte im autnen Bereich heute nicht mehr weisungsbefugt sind. Auch im Bereich der vertikalen Arbeitsteilung gilt der Vertrauensgrundsatz, da ansonsten ein arbeitsteiliges Zusammenwirken im Klinikalltag nicht möglich wäre. Der Vertrauensgrundsatz erfährt jedoch gerade bei der vertikalen Arbeitsteilung nicht unerhebliche Einschränkungen. Überträgt beispielsweise der Operateur eigene Aufgaben an ihn unterstellte Assistenten (Eröffnung des Abdomens, Wundverschluß), so ist er verpflichtet, sich über deren Qualifikation zu informieren und sie ausreichend anzuleiten bzw. zu kontrollieren. Besondere Sorgfaltspflichten ergeben sich für die unentbehrliche $\mathrm{Zu}-$ sammenarbeit des Arztes mit nicht ärztlichen Mitarbeitern. Fehlt es insoweit an der gebotenen Sorgfalt bei der Auswahl, Anleitung und Überwachung und kommt der Patient dadurch zu Schaden, so muss der Arzt für die Nachlässigkeiten und Fehler des nachgeordneten Personals nach dem Prinzip der Letztverantwortlichkeit selbst einstehen. Primär stellt sich für den Arzt in diesem Zusammenhang die Frage, welche Aufgaben überhaupt an nachgeordnetes ärztliches und nichtärztliches Personal delegiert werden dürfen. Bei einem Risikopatienten darf etwa der Anästhesiefacharzt einen AiP bzw. Berufsanfänger selbstverständlich nicht mit der selbständigen Durchführung und Überwachung der Narkose be- auftragen. Auch die Delegation einer Bluttransfusion auf nicht ärztliches Personal ist unzulässig.

Vertikale Arbeitsteilung:

Beispiele: Chefarzt - Oberarzt -

Assistenzarzt - AiP - nicht ärztliches Personal.

Haftung: Chefarzt - Oberarzt, Prinzip der Letztverantwortlichkeit.

Ob und wie weit die Mitarbeiter zu überwachen und anzuleiten sind, hängt von der jeweiligen Ausbildung, Erfahrung und Zuverlässigkeit des betreffenden Mitarbeiters ab. Sehr schwierige und anspruchsvolle Operationen können beispielsweise nicht auf einen erst am Ende der Facharztausbildung stehenden Arzt zur selbständigen Erledigung übertragen werden, und es versteht sich von selbst, dass ein Berufsanfänger überhaupt keine selbständigen Eingriffe durchführen sollte. Andere Aufgaben erfordern detaillierte Anweisungen und engmaschige Kontrollen, die auch dokumentiert werden sollten. Bestimmte Tätigkeiten, wie z.B. intravenöse Injektionen im Rahmen der Narkose können ebenfalls nicht ohne weitere Überwachung auf nicht ärztliches Personal übertragen werden, wenn nicht die entsprechenden Fachweiterbildung erfolgt ist. Dagegen ist das Vorbereiten des OP-Instrumentariums eine originäre Aufgabe der OP-Schwester bzw. des OP-Pflegers.

Im Übrigen sei darauf hingewiesen, dass die zivil- und strafrechtliche Haftung des nichtärztlichen OP-Personals in der forensischen Praxis bis heute eine eher seltene Ausnahme darstellt, da sich die zivilrechtlichen Vorwürfe üblicherweise gegen den Krankenhausträger bzw. die behandelnden Ärzte richten und auch von Seiten der Staatsanwaltschaft in $\mathrm{Er}$ mittlungsverfahren zunächst die Verantwortlichkeit des ärztlichen Personals überprüft wird. Die Problematik der zivilund strafrechtlichen Haftung des nichtärztlichen Personals wird in einem der nächsten Hefte des OP-Journals gesondert erörtert werden.

\section{Organisationspflichten des Krankenhausträgers}

Den Klinikträger treffen im Wesentlichen Organisationspflichten, wobei die Rechtsprechung insoweit hohe Anforderungen stellt. Der Träger hat Strukturen zu entwickeln und aufzubauen, die die Verantwortlichkeiten innerhalb einer Abteilung und zwischen den einzelnen Abteilungen 
regeln. Er hat den Chefarzt zu überwachen, ob er seine diesbezüglichen Pflichten wahrnimmt. Dabei reichen bloße Routinevisiten nicht aus, sondern es muss durch gezielte Kontrollen und im Einzelfall und entsprechende Dienstanweisungen zur generellen Regelung dafür gesorgt werden, dass die Arbeitsabläufe korrekt strukturiert und überwacht werden. Im operativen Bereich hat der Klinikträger daher z.B. für ein reibungsloses Hand-in-Hand-Arbeiten von Chirurgen und Anästhesisten zu sorgen. Er muss gewährleisten, dass nur geeignete Ärzte, die den Anforderungen gewachsen sind, tätig werden und dass bei der Behandlung nach dem Stand der medizinischen Wissenschaft vorgegangen wird. $\mathrm{Er}$ muss weiter sicherstellen, dass bei Operationen der Facharztstandard auch außerhalb der üblichen Arbeitszeiten im Notdienst gewährleistet wird, wobei „Facharztstandard“ nicht bedeutet, dass bei jeder Operation ein Arzt mit „Facharztpatent" anwesend ist. Grundsätzlich reicht aus, dass der betreffende Arzt den jeweiligen Eingriff bzw. die entsprechende Maßnahme, mit dem er sich befasst, so gut wie ein Facharzt beherrscht. Neben den personellen Standards müssen auch die sachlichen Standards Räumlichkeiten, apparative Ausstattung etc. - durch den Krankenhausträger sichergestellt werden. Ist dies - z. B. aus finanziellen Gründen - nicht im gebotenen Umfang möglich, verlangt die Rechtsprechung ohne wenn und aber den Verzicht auf Behandlungen, die nicht standardgerecht erbracht werden können. Bei Verletzungen der Organisationspflichten droht dem Klinikträger die Haftung wegen eines Organisationsverschuldens.

Zum Risikomanagement gehört es dabei, die Verantwortlichkeiten und Vertretungsregelungen, die Kontrollmechanismen, den Informationsfluss und die Behandlungsanweisungen schriftlich zu fixieren und entsprechend weiterzuleiten und sicherzustellen, dass das Aufklärungsgespräch ordnungsgemäß durchgeführt und dokumentiert wird. Zusätzlich hat der Krankenhausträger für die angemessene Dokumentation der Operation, der Narkose und des prä- sowie postoperativen Behandlungsverlaufes zu sorgen. Im Bereich des OP müssen selbstverständlich auch die erforderlichen Maßnahmen zur Gewährleistung des hygienischen Standards nachvollziehbar dokumentiert werden.

\section{Einsatz computergestützter Operationssysteme}

Bisher sind noch keine Haftungsrichtlinien zum Einsatz von Robotik oder Navigationssystemen entwickelt worden. Es besteht zwar eine DIN-Regelung zur Gewährleistung der Sicherheit beim Einsatz von Industrierobotern, jedoch lassen sich die darin gefassten Grundsätze nicht auf den Einsatz von Robotern im Operationssaal übertragen, da sich die Gefahrensituationen beider Technologien grundsätzlich unterscheiden. Es muss deshalb, solange keine eigenständige rechtliche Regelung besteht - eine Erstellung solcher Richtlinien wäre im übrigen sehr wünschenswert - auf die allgemeinen Grundsätze des Arzthaftungsrechts und das Medizinproduktegesetz zurückgegriffen werden. Der Einsatz von Navigationssystemen oder Operationsrobotern setzt die sehr ausführliche Aufklärung des Patienten, eine exakte Vorbereitung und Durchführung der Operation sowie die besonders sorgfältige und vollständige Dokumentation des Eingriffs voraus. Auch wenn heute sicherlich noch keine Verpflichtung angenommen werden kann, Navigationssysteme oder gar Robotik einzusetzen oder dem Patienten anzubieten, muss insbesondere bei bestimmten Indikationen (Navigation beim Einbringen der Pedikelschrauben und bei der Positionierung der Bohrkanäle bei der Kreuzbandersatzplastik etc.) damit gerechnet werden, dass die Rechtsprechung nach entsprechender Beratung durch medizinische Sachverständige - die Forderung aufstellen wird, dass der Patient über derartige Möglichkeiten zumindest informiert werden muss, um anschließend selbstbestimmt entscheiden $\mathrm{zu}$ können.

\section{Aufklärung}

Da sich die meisten, auf dem Markt befindlichen Systeme noch in der Phase der klinischen Erprobung befinden, ist insbesondere auf eine umfassende und vollständige Aufklärung zu achten, die grundsätzlich schriftlich festzuhalten ist, wobei Formblätter - soweit sie überhaupt schon vorhanden sind - allein vermutlich nicht ausreichen werden. Bei neuen, risikoreichen Eingriffen, stellt die Rechtsprechung sehr hohe Anforderungen an die Aufklärung, um das Selbstbestimmungsrecht des Patienten zu wahren. Grundsätzlich muss jeder Patient zunächst auf die Freiwilligkeit einer Studienteilnahme hingewiesen werden. Es ist erforderlich, dass der Patient über den Studiencharakter der Versorgung informiert wird und explizit sein Einverständnis in das beabsichtigte Verfahren gibt. Dies ist vor allem dann notwendig, wenn das Verfahren hinsichtlich der speziellen verfahrensimmanenten Risiken für den Patienten - und auch für die Ärzte - noch relativ unbekannt ist und eine erhöhte Gefahr durch das neue Verfahren nicht sicher ausgeschlossen werden kann. Weiterhin ist es bei der Anwendung von Operationsrobotern im Rahmen klinischer Studien aufgrund der Pflicht zur Gewährleistung eines standardisierten Studienablaufs und zur Qualitätssicherung notwendig, sämtliche Befunde standardisiert zu erfassen und engmaschig Nachuntersuchungstermine zu planen.

Daneben ist der Patient - auch wenn das Verfahren bereits routinemäßig eingesetzt wird - neben den allgemeinen Operationsrisiken über sämtliche operationstypischen Gefahrentatbestände und Behandlungsalternativen, sowie Vor- und Nachteile der jeweiligen Verfahren zu unterrichten. Der Zeitpunkt der Aufklärung ist bei Neulandoperationen so früh wie möglich anzusetzen, um den Patienten ausreichend Überlegungszeit ggf. auch für eine Rücksprache oder das Einholen einer weiten Meinung zu geben.

\section{Aufklärung:}

Über Chancen, Risiken und Folgen der gewählten Behandlung und über mögliche Alternativen muss umso intensiver aufgeklärt werden, je größer das Behandlungsrisiko ist, je gravierender die Gesundheitsschäden im Falle des Eintritts des Behandlungsrisikos sind, je umstrittener die gewählte Behandlungsmethode innerhalb der Medizin ist, je mehr Behandlungsalternativen bestehen, je verzichtbarer die fragliche Behandlung ist.

\section{Indikationsstellung}

Entscheidend ist zunächst die richtige Indikationsstellung zum Einsatz einer roboterassistierten Operation. Bei jeder ärztlichen Behandlung ist es geboten, den größtmöglichen Nutzen für den $\mathrm{Pa}$ tienten bei geringst möglichen Belastungen zu gewährleisten. Ein Behandlungsfehler kann deshalb auch dann vorliegen, wenn unter mehreren in Frage kommenden Therapiemöglichkeiten eine risikoreichere angewandt wird.

Insbesondere Neulandmethoden müssen sorgfältig mit konkurrierenden Verfahren der Schulmedizin verglichen und gegen- 
einander abgewogen werden. Eine Behandlung mit neuen Techniken muss deshalb zumindest den Nutzen versprechen, den die Standardbehandlung im Normalfall gewährleistet, wobei der behandelnde Arzt auch das Standardverfahren beherrschen muss, um ggf. umsteigen zu können, wenn sich gravierende Probleme ergeben. Grundsätzlich ist bei jedem Einzelfall zu prüfen, ob nicht eine andere therapeutische Methode - vor allem wenn es einen „goldenen Standard“ gibt - bevorzugt werden sollte. Jede Neulandbehandlung ist ein Abweichen vom Standard. Weicht ein Arzt hiervon $a b$, dann hat er im Haftungsfall darzulegen, warum die Abweichung von der konventionellen Methode erforderlich bzw. wenigstens gerechtfertigt war. Selbstverständlich ist auch, dass nicht jeder Patient für die Behandlung mit neuen Verfahren geeignet ist. Besondere Vorsicht ist bei minderjährigen, dementen oder aus anderen Gründen nicht einwilligungsfähigen Patienten geboten, bei denen Navigation bzw. Robotik zumindest heute noch nur in Ausnahmefällen sehr zurückhaltend eingesetzt werden sollte.

Richtige Indikation:

Abwägung gegenüber anderen

(Standard-)Verfahren,

sachgerechte Auswahl der Patienten.

\section{Durchführung der Operation}

Aus den oben genannten Grundsätzen lässt sich ableiten, dass sämtliche Sicherheitssysteme und Steuerungseinheiten des Roboters eine zusätzliche Gefährdung oder Fehlbehandlung des Patienten über das Normalmaß hinaus ausschließen sollten. Es muss gewährleistet sein, dass auch für den Fall des kompletten Ausfalls des Robotersystems die Operation auf konventionelle Weise fachgerecht ohne zeitliche Verzögerung fortgeführt werden kann. Dies bedeutet, dass der Operateur bzw. das Operationsteam sowohl die konventionelle Operationstechnik, als auch den Umgang mit dem Operationsroboter beherrschen muss. Neben der korrekt funktionierenden Maschine und der operativen Kenntnis muss das behandelnde ärztliche und nichtärztliche Personal auch in der Lage sein, den Roboter bzw. das Navigationssystem korrekt zu warten und eventuell auftretende Fehler rechtzeitig zu erkennen.

\section{Komplikationen}

Es darf sich aus dem Einsatz des Operationsroboters keine Verschlechterung des Operationsergebnisses für den Patienten ergeben. Es muss darauf geachtet werden, dass es durch den Einsatz des Robotersystems zu keiner Zunahme von Komplikationen kommt. Stellt sich z.B. heraus, dass es unter Einsatz einer neuen Methode zu einer erhöhten Blutungsgefahr während der Operation oder zu einem vermehrten Auftreten von postoperativen Wundheilungsstörungen aufgrund von Infektionen kommt, ist der Einsatz der neuen Methode entsprechend $\mathrm{zu}$ modifizieren oder im Zweifel zu unterlassen. Außerdem trifft den Arzt und den Krankenhausträger die Pflicht, sich bei Schwierigkeiten über den Träger der Herstellerfirma um Hilfe zu bemühen.

\section{Dokumentation}

Nicht zu vernachlässigen ist in diesem Zusammenhang, auf eine umfassende Dokumentation zu achten, da Unzulänglichkeiten in der Dokumentation im gerichtlichen Verfahren zu Beweiserleichterungen für den Patienten bis hin zur Beweislastumkehr im Schadensprozess führen können. Dokumentiert werden müssen sämtliche erhobenen Befunde und therapeutischen Schritte, aber auch Komplikationen und insbesondere die Aufklärung des Patienten über die Behandlung, die Risiken der Behandlung und Behandlungsalternativen. Moderne Maschinen sollten selbst für eine ausreichende Dokumentation der von ihnen ausgeführten Arbeitsschritte sorgen.

\section{Koordination und Kommunikation}

Die Tatsache, dass beim Einsatz der Robotertechnik verschiedene Fachrichtungen der Medizin - Chirurgie bzw. Orthopädie, Radiologie und Anästhesie - ineinandergreifen, macht es notwendig, dass diese Zusammenarbeit reibungslos funktioniert und dass an den Schnittstellen keine Übertragungsfehler entstehen. Koordiniert der Operateur die Planung des Eingriffs einschließlich aller vorbereitenden Maßnahmen, so ist er letztlich auch für das Zusammenwirken der verschiedenen Fachbereiche verantwortlich. Ist die Vorbereitung und Planung nicht auf den Operateur übertragen, sondern wird dies von einem Dritten organisiert, dann ergeben sich hier zwei voneinander getrennte Verantwortlichkeiten.
Für das Gelingen der Operation an sich haftet grundsätzlich der die Operation ausführende Chirurg oder Orthopäde selbst. Er hat die Operation nach den Regeln der ärztlichen Sorgfalt seines Fachgebiets durchzuführen. Vor Beginn der Operation muss er die Funktionstüchtigkeit des assistierenden Operationsroboters überprüfen und gegebenenfalls geeignete Maßnahmen treffen, um diese wieder herzustellen.

Der das für die Operationsplanung notwendige CT erstellende Radiologe ist für die fehlerfreie Erfassung und Verarbeitung der von ihm ermittelten Daten verantwortlich.

Grundsätzlich darf sich der Operateur darauf verlassen, dass ein Kollege anderer Fachrichtung die in seinen Aufgabenbereich fallende Untersuchungsmethoden und -ergebnisse fehlerfrei durchgeführt und erstellt hat. Jedoch hat der Operateur die gewonnenen Daten, wie bereits ausgeführt, auf ihre Plausibilität hin zu überprüfen. Sollten sich dabei offensichtliche Mängel ergeben, so hat der Operateur diese zu beseitigen.

In den Verantwortungsbereich des Anästhesisten fällt die vollständige Relaxierung des Patienten während des Einsatzes des Operationsroboters. Jedoch muss der Operateur darauf achten, dass im Falle einer reflexartigen Bewegung des Patienten eine Neueinstellung des Systems oder sogar der Umstieg auf ein konventionelles Verfahren erforderlich sein kann.

Verantwortlichkeiten:

Operateur: Planung und Durchführung der Operation.

Anästhesist: Narkose, insbesondere ausreichende Relaxation. Radiologe: Erstellung und Befundung des CT.

\section{Qualitätsmängel}

Qualitätsmängel von Navigationssystemen oder Robotikgeräten sind für den Operateur in aller Regel nicht kontrollierbar. Ihn trifft lediglich die Pflicht, sich mit der Funktionsweise so weit vertraut zu machen, wie dies einem naturwissenschaftlich und technisch aufgeschlossenen Menschen möglich und zumutbar ist. Er haftet daher auch insoweit nicht für Mängel der neuen Technik, welche in einem reinen nicht vorhersehbaren Geräteversagen liegen. Eine Gefährdungshaftung ohne Verschulden existiert 
insoweit nicht. Sowohl der Operateur als auch der Krankenhausträger sind aber verpflichtet, die weitere Entwicklung auf diesem Gebiet, sorgfältig zu beobachten. Ein Ausgleich für den Patienten ergibt sich aus der Haftung des Produzenten nach dem Produkthaftungsgesetz bzw. nach allgemeinem Deliktsrecht. Nach dem Produkthaftungsgesetz unterliegt der Hersteller eines Produktes für Herstellungsfehler einer verschuldensunabhängigen Ersatzpflicht. Hersteller ist, wer das Endprodukt, einen Grundstoff oder ein Teilprodukt hergestellt hat. Dies kann im Einzelfall auch der Arzt sein, welcher sich an der Entwicklung der neuen Technik beteiligt. Die Hersteller von Navigationssystemen und Robotikgeräten können für Materialfehler, aber auch für Fehler, die in der Fertigung des Gerätes liegen, verantwortlich gemacht werden. Die Haftung nach allgemeinem Deliktsrecht, bei der es sich um eine verschuldensabhängige Produzentenhaftung handelt, ist insbesondere dann von Bedeutung, wenn es sich um eine Verletzung von Produktbeobachtungspflichten, sog. Entwicklungsrisiken, handelt, die nach dem Produkthaftungsgesetz nicht gedeckt sind.

Qualitätsmängel:

Haftung des Produzenten, da Qualitätsmängel in der Regel für den Operateur nicht überprüfbar sind.

\section{Hygiene und Desinfektion}

Das Krankenhaus hat alle notwendigen Maßnahmen zu treffen, die für die Erkennung, Verhütung und Bekämpfung von nosokomialen Infektionen erforderlich sind. Dies gilt insbesondere für die OP-Hygiene und die Klimatisierung des Operationssaals. Daneben müssen die baulichen Verhältnisse des Operationstrakts (OP-Schleusen, Operationssäle, etc.) sowie die durchzuführenden Desinfektionsmaßnahmen (Instrumentarium,
Flächendesinfektion) dem heute anerkannten hygienischen Standard entsprechen. Hierzu wurden in den letzten Jahren Hygieneverordnungen auf der Grundlage der jeweiligen Krankenhausgesetze erlassen, die als Richtlinien für den geforderten Standard anzusehen sind.

Da die meisten Infektionen bei Operationen durch Keime ausgelöst werden, die vom Personal oder vom Patienten selbst stammen, kommt dem adäquaten Verhalten des OP-Personals und einer entsprechenden Schutzkleidung entscheidende Bedeutung zu. Der Operateur hat dafür Sorge zu tragen, dass sämtliche an der Operation beteiligten Mitarbeiterinnen und Mitarbeiter die geltenden Hygienevorschriften einhalten, insbesondere Schutzkleidung und Gesichtsmasken tragen. Daneben ist der Operateur auch für ein möglichst atraumatisches und zügiges Vorgehen bei der Operation selbst verantwortlich.

Verantwortlichkeiten:

Klinikträger: OP-Hygiene, Desinfektion des Instrumentariums, bauliche Substanz.

Operateur: Steriles Vorgehen bei der Operation selbst, Anleitung und Überwachung der an der Operation beteiligten Mitarbeiter.

\section{Literatur}

1 Bundesgerichtshof in Zivilsachen. BGHZ 140: $309-319$

${ }^{2}$ Giesen D. Arzthaftungsrecht. Mohr JCB (Paul Siebeck), Tübingen 1995

${ }^{3}$ Heinze M, Jung jun H. Die haftungsrechtliche Eigenverantwortlichkeit des Krankenpflegepersonals in Abgrenzung zur ärztlichen Tätigkeit. MedR 1985; 3: 62 -71

${ }^{4}$ Laufs A, Uhlenbruck W, Genzel H et al. Handbuch des Arztrechts. 2. Auflage. $\mathrm{CH}$ Beck'sche Verlagsbuchhandlung. München 1999

${ }^{5}$ Laufs A. Arztrecht. NJW-Schriftenreihe. 5. Auflage. CH Beck'sche Verlagsbuchhandlung. München 1993

${ }^{6}$ Rudolph H. OP-Kleidung und Patientenabdeckung. OP-Journal 1993; 9: $72-74$
${ }^{7}$ Stegers C-M. Vom Organisationsmangel zum Risikomanagement. MedR 1997; 15: 390 396

${ }^{8}$ Stindt J. Haftungsrechtliche Relevanz von Organisationsstrukturen. In: Arbeitsgemeinschaft Rechtsanwälte im Medizinrecht e.V. (eds.): Krankenhaus im Brennpunkt. Springer Verlag, Berlin, Heidelberg 1997; S $27-35$

${ }^{9}$ Wilhelm D. Probleme der medizinischen $\mathrm{Ar}-$ beitsteilung aus strafrechtlicher Sicht. MedR 1983; $1: 45-51$

${ }^{10}$ Wille B. Epidemiologie, Infektiologie und Verhütungsmaßnahmen postoperativer Wundinfektionen. OP-Journal 1996; 12: $91-93$

${ }^{11}$ Rumler-Detzel P. Budgetierung - Rationalisierung - Rationierung - Einflüsse auf die medizinische Leistungsfähigkeit oder Senkung des medizinischen Standards? VersR 1998; S. 546

12 OLG Oldenburg. VersR 1995; S. 49

${ }^{13}$ BGH. VersR 1983; S. 244

${ }^{14}$ BGH. VersR 1974; S. 804

${ }^{15}$ Bergmann KO. Die Organisation des Krankenhauses unter haftungsrechtlichen Gesichtspunkten. VersR 1996; S. 810

${ }^{16}$ Kleinewefers H. Zur zivilrechtlichen Haftung des Arztes - Ärztliche Verantwortung, Grundfragen und Beispiele. VersR 1992 1425

17 OLG Karlsruhe. VersR 1989; 195

\section{Dr. med. H. Hölzer}

Rechtsanwalt, Facharzt für Chirurgie

KHWP - Rechtsanwälte und Notare

Uhlandstr. 11

70182 Stuttgart

Dr. med. cand. iur. H. Striegel

Med. Klinik und Poliklinik,

Abt. Sportmedizin

Universität Tübingen

Hölderlinstr. 11

72074 Tübingen

\section{Winter}

Rechtsanwalt

KHWP - Rechtsanwälte und Notare

Uhlandstr. 11

70182 Stuttgart 